\title{
Lesions of the Basolateral Amygdala Disrupt Conditioning Based on the Retrieved Representations of Motivationally Significant Events
}

\author{
Dominic M. Dwyer and Simon Killcross \\ School of Psychology, Cardiff University, Cardiff CF10 3YG, United Kingdom
}

\begin{abstract}
One recent perspective (Blundell et al., 2001; 2003; Killcross and Blundell, 2002; Balleine et al. 2003) on the function of the basolateral region of the amygdala (BLA) suggests that it plays an important role in the representation of the sensory features of motivationally significant events. This predicts that lesions of the BLA will not produce a decrement in performance in conditioning procedures based on the formation of associations between the sensory aspects of neutral events but will interfere with conditioning based on associations between neutral cues and motivationally significant events. This prediction is supported by the evidence that BLA lesions were without effect on a sensory preconditioning procedure (experiment 1A) that used neutral cues but that BLA lesions did significantly impair representation-mediated conditioning (experiment $1 \mathrm{~B}$ ) when the target cues were motivationally significant at the time of training. These results demonstrate that animals with lesions of the BLA can represent the sensory aspects of neutral events but not the sensory aspects of motivationally significant events.
\end{abstract}

Key words: association; basolateral amygdala; mediated conditioning; classical conditioning; motivation; sensory preconditioning

\section{Introduction}

The effects of selective lesions of the amygdala have been investigated in a variety of Pavlovian conditioning procedures although they do not appear to prevent basic conditioning. For example, animals with BLA lesions were able to learn to approach a food magazine during the presentation of a light conditioned stimulus (CS) that signaled food delivery (Hatfield et al., 1996), and this type of Pavlovian conditioned response (CR) has been observed in animals with lesions of the BLA in a number of similar procedures (Blundell et al., 2001; Setlow et al., 2002). A related finding is that animals with lesions of the BLA were able to learn a signtracking response in a Pavlovian autoshaping task (Blundell et al., 2003). Furthermore, the acquisition of basic taste aversions is not impaired in animals with BLA lesions (Dunn and Everitt, 1988; Blundell et al., 2003; but see Morris et al., 1999).

However, even in tasks in which BLA lesions did not prevent the acquisition of a basic Pavlovian response, they were not totally without effect. In particular, although BLA-lesioned animals are able to learn to approach a food magazine during the presentation of a CS that had been paired with food, unlike shamlesioned animals, they showed no reduction in this magazine CR when the food unconditioned stimulus (US) was subsequently devalued by pairing with lithium chloride ( $\mathrm{LiCl}$ )-induced nausea

\footnotetext{
Received April 18, 2006; revised July 5, 2006; accepted July 8, 2006.

This work was supported by a Medical Research Council Career Establishment Grant 69805345 (S.K.).

Correspondence should be addressed to Dominic M. Dwyer, School of Psychology, Tower Building, Cardiff University, Cardiff CF10 3YG, UK. E-mail: DwyerDM@cardiff.ac.uk.

D0I:10.1523/JNEUROSCI.1647-06.2006

Copyright $\odot 2006$ Society for Neuroscience $\quad$ 0270-6474/06/268305-05\$15.00/0
}

(Hatfield et al., 1996). A similar deficit has been observed in a Pavlovian autoshaping procedure in which devaluation of a US with a specific satiety treatment selectively reduced responding to the CS associated with that US in sham-, but not BLA-lesioned, rats (Blundell et al., 2003).

On the basis of these results and others from instrumental conditioning procedures (Blundell et al., 2001; Balleine et al., 2003; Corbit and Balleine, 2005), it was suggested that the BLA has an important role in the representation of the unique sensory aspects of motivationally significant stimuli (Killcross and Blundell, 2002; Balleine et al., 2003; Blundell et al., 2003). In particular, the suggestion was made that lesions of the BLA would disrupt "the use of CS-evoked representations of the unique sensory or hedonic effects of motivationally significant events, but not the more general reinforcing aspects" (Blundell et al., 2003). One prediction of this account is that BLA-lesioned animals will not show impaired performance on conditioning tasks that involve learning about neutral stimuli. However, BLA lesions should impair performance on tasks that involve the CS-evoked representation of a motivationally significant stimulus in learning. Run in parallel, experiments $1 \mathrm{~A}$ and $1 \mathrm{~B}$ examined the performance of BLA-lesioned animals on performance in a sensory preconditioning task and a mediated conditioning task, respectively. The sensory preconditioning procedure relied on associations between neutral stimuli, whereas the mediated conditioning procedure relies on the CS-evoked representation of motivationally significant stimuli. According the preceding analysis, lesions of the BLA would be expected to interfere with performance in experiment $1 \mathrm{~B}$ but not in experiment $1 \mathrm{~A}$. 


\section{Materials and Methods}

\section{Subjects and apparatus}

Sixteen male hooded Lister rats (OLAC, Bicester, UK) were used in each experiment. They were housed in pairs in a temperature- and humiditycontrolled room, which was lit between 8:00 A.M. and 8:00 P.M. Rats were maintained on ad libitum food and water until being operated on when weighing at least $300 \mathrm{~g}$. After surgery, they were returned to their home cages for a period of $10-14 \mathrm{~d}$ recuperation. The rats used in experiment $1 \mathrm{~A}$ were subsequently placed on a $22.5 \mathrm{~h}$ water-deprivation schedule under which they received water for $1.5 \mathrm{~h}$ each day. This access was given in the home cage $2 \mathrm{~h}$ after experimental fluid access had taken place. The rats used in experiment $1 \mathrm{~B}$ were subsequently placed on a $22.5 \mathrm{~h}$ food and water deprivation schedule, under which they received both food and water for $1.5 \mathrm{~h}$ each day. This access was given in the home cage $2 \mathrm{~h}$ after behavioral testing had taken place. Throughout both experiments, fluid consumption was assessed by weighing drinking bottles before and after consumption.

In experiment $1 \mathrm{~A}$, the solutions presented were $5 \%(\mathrm{w} / \mathrm{v})$ sucrose, $5 \%$ (w/v) maltodextrin, $0.01 \mathrm{M} \mathrm{HCl}$, and $60 \mu \mathrm{M}$ quinine hydrochloride, or compounds comprised of these elements with the concentrations maintained. Fluid access during training and testing took place in eight feeding cages in a room separate from the rats' home cages. These were molded plastic boxes (similar to, although smaller than, the rats home cages) $30 \times 13 \times 11 \mathrm{~cm}$ in size, with a wire mesh ceiling. The spouts of two drinking bottles could be inserted through the wire mesh ceilings.

In experiment $1 \mathrm{~B}$, the solutions presented were water, $15 \%(\mathrm{w} / \mathrm{v}) \mathrm{su}-$ crose, and $15 \%(\mathrm{w} / \mathrm{v})$ maltodextrin. Behavioral training took place in a $\mathrm{Y}$ maze which was modified so that rats could be confined to one arm at a time. All arms were of the same dimensions $(20 \times 12.5 \times 36 \mathrm{~cm})$, had a wire mesh ceiling, and a hole in the end wall through which the spout of a drinking bottle could be inserted. The three arms were made distinct by the addition of colored walls and textured floors. One arm had white walls and sandpaper floor, a second had black walls and a black rubber floor, and the third arm has plain aluminum walls and floors. The maze was placed in the center of a square room, directly under the single light, and remained in the same orientation throughout. The presence of a door in one wall, a video camera mounted opposite the door, along with colored posters on the remaining two walls served as distal cues for the orientation of the maze. During training, the arms were separated with aluminum dividers that restricted access to the one arm, plus the central choice section of the Y maze. Despite being prevented from accessing them by these dividers, rats were able to see the other arms as well as the distal cues behind them. During place-preference testing the dividers were removed allowing the rats free access to all three arms. Flavorpreference testing took place in the feeding cages described in experiment $1 \mathrm{~A}$.

\section{Surgical procedures}

Rats were anesthetized using an isoflurane/oxygen mix, their heads shaved, and placed in a stereotaxic frame (David Kopf, Tujunga, CA) with the incisor bar set to $-3.3 \mathrm{~mm}$. An incision was made to expose the skull and the position of Bregma was noted. Rats in group BLA ( $n=8$ for both of experiments $1 \mathrm{~A}$ and $1 \mathrm{~B}$ ) received intracranial injections of $0.09 \mathrm{M}$ quinolinic acid dissolved in phosphate buffer at the following coordinate sites: anteroposterior, -2.3 and $-3.0 \mathrm{~mm}$; lateral, $\pm 4.6 \mathrm{~mm}$ (all from Bregma); ventral, $-7.3 \mathrm{~mm}$ (from Dura). The injections were made using a $1 \mu \mathrm{l}$ Hamilton syringe (SGE, Ringwood, Victoria, Australia) attached to a microinjector (David Kopf, Tujunga, CA). $0.3 \mu$ l of quinolinic acid was injected over $3 \mathrm{~min}$ at each of the four sites and the needle left in place for $5 \mathrm{~min}$ to allow diffusion of the toxin. To control for the nonspecific effects of surgery, rats in group sham $(n=8$ for both of experiments $1 \mathrm{~A}$ and $1 \mathrm{~B}$ ) received the same surgical procedure with the exception that the syringe was filled with phosphate buffer alone and no fluid injected.

\section{Histological procedures}

No longer than $7 \mathrm{~d}$ after the completion of behavioral procedures, rats were injected with a lethal barbiturate overdose and perfused transcardially with $0.9 \%$ saline followed by $10 \%$ formalin solution. The brains were stored in $10 \%$ formalin solution for at least $48 \mathrm{~h}$ before being transferred to a $25 \%$ sucrose solution. The brains were then frozen and $40 \mu \mathrm{m}$ coronal sections were cut throughout the region of the BLA, mounted on glass slides, and stained with cresyl violet. Slides were examined for placement and extent of the lesion. The latter was assessed by microscopically examining sections of lesioned, as compared with sham, brains for the following features: gross morphological changes such as holes and tissue collapse, gliosis and scarring, and signs of neuronal cell body shrivelling and loss.

\section{Behavioral procedures: experiment $1 \mathrm{~A}$}

Two days after being placed on the water deprivation schedule, behavioral training began with $8 \mathrm{~d}$ of exposure to two compound stimuli, $\mathrm{AX}$ and BY, where A and B were counterbalanced between sucrose and maltodextrin, and $\mathrm{X}$ and $\mathrm{Y}$ counterbalanced between quinine hydrochloride and $\mathrm{HCl}$. Throughout acquisition, rats were allowed $30 \mathrm{~min}$ access to 10 $\mathrm{ml}$ of the relevant solution. On days $1,3,5$, and 7, half of the rats received the AX compound, and on days $2,4,6$, and 8 they received BY. The remaining rats received $\mathrm{BY}$ on the odd number days and $\mathrm{AX}$ on the even numbered days. On days 9 and 12, all rats received flavor $\mathrm{X}$ followed by an intraperitoneal injection of $0.15 \mathrm{M} \mathrm{LiCl}$ at $20 \mathrm{ml} / \mathrm{kg}$. On days 11 and 14 they received flavor $Y$ without consequences, whereas on days 10 and 13 they received water.

Flavor aversion testing occurred over the next $2 \mathrm{~d}$. On day 14, all rats received a $30 \mathrm{~min}$ choice test between maltodextrin and sucrose (flavors $\mathrm{A}$ and B). On day 15, all rats received a $30 \mathrm{~min}$ choice test between quinine hydrochloride and $\mathrm{HCl}$ (flavors $\mathrm{X}$ and $\mathrm{Y}$ ).

\section{Behavioral procedures: experiment $1 B$}

Acquisition. Four days after being placed on the food and water deprivation schedule, behavioral training began with $9 \mathrm{~d}$ of exposure to the three arms of the Y maze. On days 1, 4, and 7, all rats were exposed to one arm (henceforth the neutral arm) for $15 \mathrm{~min}$ and allowed to consume a maximum of $15 \mathrm{ml}$ of water. On days 2, 5, and 8, all rats were exposed to a second arm (henceforth the target arm) for $15 \mathrm{~min}$ and allowed to consume a maximum of $15 \mathrm{ml}$ of either sucrose or maltodextrin. On days 3 , 6 , and 9, all rats were exposed to the third arm (henceforth the nontarget arm) for $15 \mathrm{~min}$ and allowed to consume a maximum of $15 \mathrm{ml}$ of whichever of the sucrose or maltodextrin they had not consumed in the target arm. The solution presented in the target arm will be referred to as the $\mathrm{CS}+$, whereas the solution presented in the nontarget arm will be referred to as the CS-. The identity of the arms was counterbalanced within groups, as was the solution consumed in the target and nontarget arms. On day 10, all rats were placed in the target arm for $15 \mathrm{~min}$, allowed to consume a maximum of $15 \mathrm{ml}$ of water, and received a intraperitoneal injection of $0.15 \mathrm{M} \mathrm{LiCl}$ at $20 \mathrm{ml} / \mathrm{kg}$ immediately after removal. On day 11 , all rats were placed in the feeding cages for $15 \mathrm{~min}$ and allowed to consume water.

Flavor-aversion and place-preference testing. Flavor-aversion testing took place over the next $2 \mathrm{~d}$. On day 12, rats were placed in the feeding cages and allowed $15 \mathrm{~min}$ access to either sucrose or maltodextrin. On day 13 they received $15 \mathrm{~min}$ access to the flavor not presented the previous day. Finally, on day 14, all rats received a place-preference test in which they were given 5 min free access to the three arms of the Y maze. All rats were started in their target arm and the sessions videotaped. These were later scored for time spent in the nontarget and neutral arms, defined as having all four paws within that particular arm.

\section{Results \\ Histology}

Figure 1 illustrates the largest and smallest lesioned areas for rats in group BLA from experiments $1 \mathrm{~A}$ and $1 \mathrm{~B}$ as well as a photomicrograph of a representative lesion. There were no recovery problems or sustained weight loss after surgery. There was significant bilateral damage to the lateral and basal nuclei of the amygdala in all lesioned rats but one animal, which was removed from the analysis of experiment 1A. Two rats were omitted from the analysis of experiment $1 \mathrm{~B}$ because of having bilateral damage to the central nucleus of the amygdala. In the remaining rats, all but the 

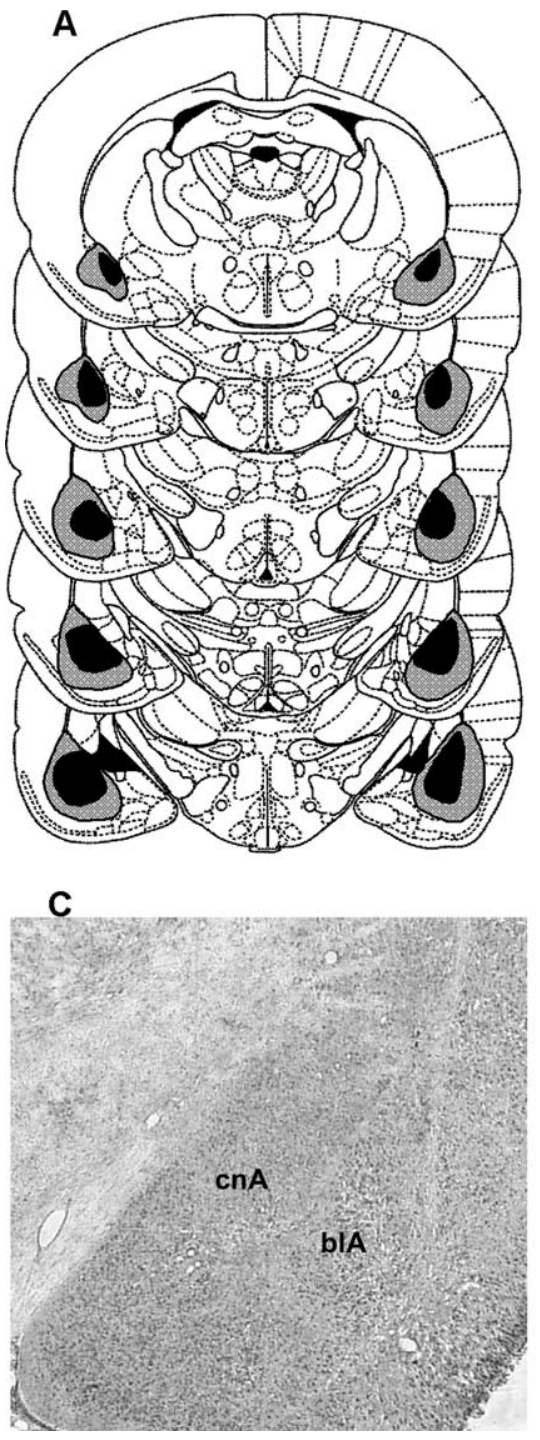
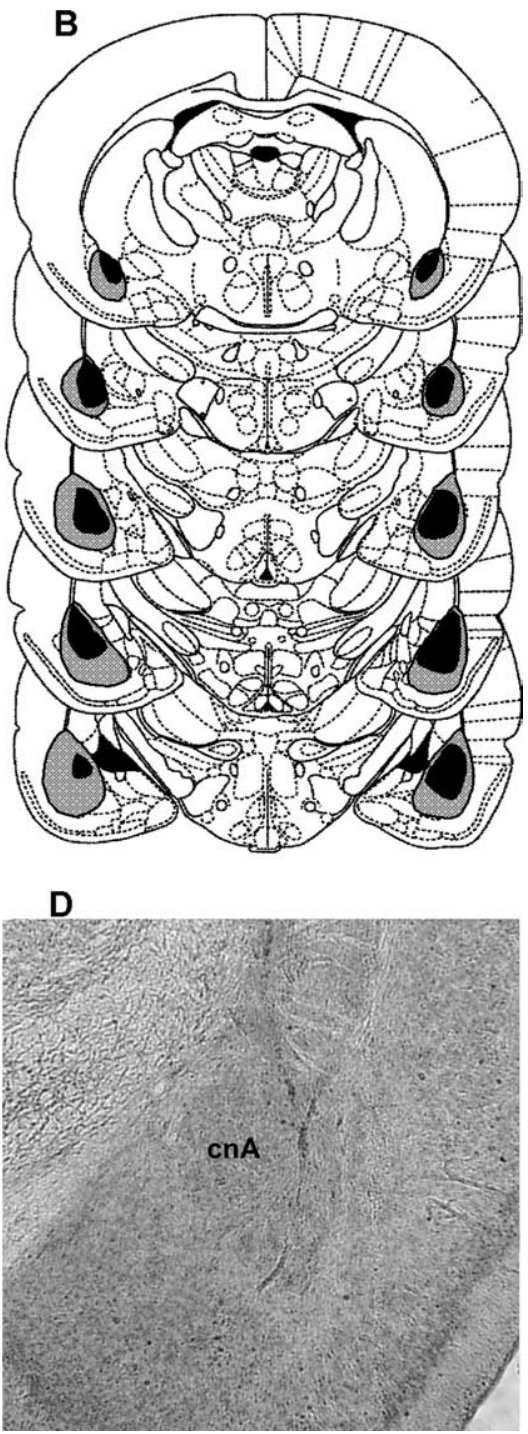

Figure 1. Schematic representation of the minimum (black) and maximum (gray) extent of damage to the BLA in experiments $1 \mathrm{~A}(\boldsymbol{A})$ and $1 \mathrm{~B}(\boldsymbol{B})$. Coronal sections are based on Paxinos and Watson (1998) and range from -1.6 to $-3.6 \mathrm{~mm}$ posterior to bregma. $\boldsymbol{C}$, A photomicrograph from a sham-lesioned animal for comparison with a representative lesioned animal (D). Note the intact central nucleus of the amygdala (cnA) in both $\boldsymbol{C}$ and $\boldsymbol{D}$ and the absence of the lateral and basolateral nuclei (b/A) from $\boldsymbol{D}$.

was used. There was a significant effect of flavor $\left(F_{(1,13)}=9.08\right)$ but none of lesion $\left(F_{(1,13)}=2.80\right)$, and no interaction between these two factors $(F<1)$. The lack of an effect of BLA lesions on sensory preconditioning was matched by a lack on an effect on the directly conditioned aversion. The right panel in Figure 2 shows the mean consumption of flavors $\mathrm{X}$ and $\mathrm{Y}$ during preference testing. Both BLA- and sham-lesioned animals consumed less of flavor $\mathrm{X}$, which was paired with $\mathrm{LiCl}$, than flavor Y, which was not, although there was a tendency for consumption in the BLA animals to be slightly greater overall. This conclusion was supported by a mixed ANOVA analysis with between-subjects factors of lesion and a within-subjects factor of flavor (X or Y). There were significant effects of flavor $\left(F_{(1,13)}=69.68\right)$ and of lesion $\left(F_{(1,13)}=4.90\right)$, but no interaction between these two factors $(F<1)$.

\section{Experiment 1B, flavor aversion}

The left panel in Figure 3 shows the mean total consumption of CS + and CS - flavors during the preference tests in BLAand sham-lesioned rats. The shamlesioned rats show a rejection of the CS+ not seen in the BLA-lesioned rats. As such, BLA lesions appear to prevent representation-mediated flavor aversion learning. This conclusion was supported by a mixed ANOVA analysis with between-subjects factors of lesion and a within-subjects factor of $\mathrm{CS}$ (CS+ vs CS-). There was a significant effect of CS $\left(F_{(1,12)}=7.06\right)$ but none of lesion $(F<1)$, as well as a significant interaction between these two factors $\left(F_{(1,12)}=6.49\right)$. Analysis of the simple effects of the CS by lesion interaction revealed that there was a significant difference between consumption of the target and nontarget flavors in the

largest lesions spared the central nucleus entirely and in no case was there bilateral damage. Lesion volumes were evaluated by submitting lesion traces to analysis with ImageJ (Abramoff et al., 2004). Lesion volumes ranged between 84 and $98 \%$ and 79 and $98 \%$ in experiments $1 \mathrm{~A}$ and $1 \mathrm{~B}$, respectively. There was also some evidence of damage to the dorsal endopiriform nucleus and some ventricular enlargement was observed at the most caudal extent of the lesions, but this was not systematic across rats.

\section{Experiment 1A, flavor aversion}

The left panel in Figure 2 shows the mean consumption of flavors $\mathrm{A}$ and $\mathrm{B}$ during the preference test in BLA- and sham-lesioned animals. Both BLA- and sham- lesioned animals show a rejection of the target flavor A, which had been pre-exposed in compound with a flavor (X) directly paired with $\mathrm{LiCl}$. As such, BLA lesions do not appear to prevent sensory preconditioning. This conclusion was supported by a mixed ANOVA analysis with betweensubjects factors of lesion and a within-subjects factor of flavor (A or B). In all the analyses reported here, a type I error rate of 0.05 sham-lesioned rats $\left(F_{(1,12)}=15.80\right)$, but not in the lesioned rats $(F<1)$. There was also a significant difference between lesioned and sham-lesioned rats in consumption of the target flavor $\left(F_{(1,12)}=5.25\right)$, but not nontarget flavor $\left(F_{(1,12)}=1.07\right)$.

\section{Experiment 1B, place preference}

The right panel in Figure 3 shows the mean time spent in the nontarget and neutral arms of the Y maze for sham- and BLAlesioned rats during the place-preference test. Both BLA- and sham-lesioned rats showed a preference for the nontarget arm (which had been paired with either sucrose or maltodextrin but not $\mathrm{LiCl}$ ) over the neutral arm (which had been paired with water). As such, BLA lesions appear to have no effect on the ability of a nutritious flavor to create a place preference. This conclusion was supported by a mixed ANOVA analysis, with betweensubjects factor of lesion and a within-subjects factor of arm (neutral or nontarget). There was a significant effect of $\operatorname{arm}\left(F_{(1,12)}=\right.$ 15.42) but not of lesion $(F<1)$, nor was there a significant interaction between these two factors $(F<1)$. 

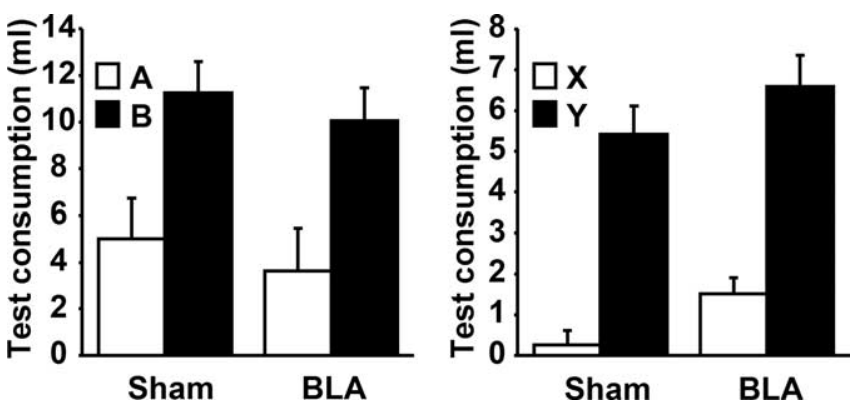

Figure 2. Mean consumption (with SEM) of the $A$ and $B$ solutions (left) as well as the $X$ and $Y$ solutions (right) during the two-bottle preference for both sham- and BLA-lesioned rats in experiment $1 A$. AX and BY had been presented as compounds during pretraining before $X$ was directly paired with $\mathrm{LiCl}$. Thus, the contrast between $A$ and $B$ represents a test of sensory preconditioning and the contrast between $X$ and $Y$ a test of directly conditioned taste aversion.
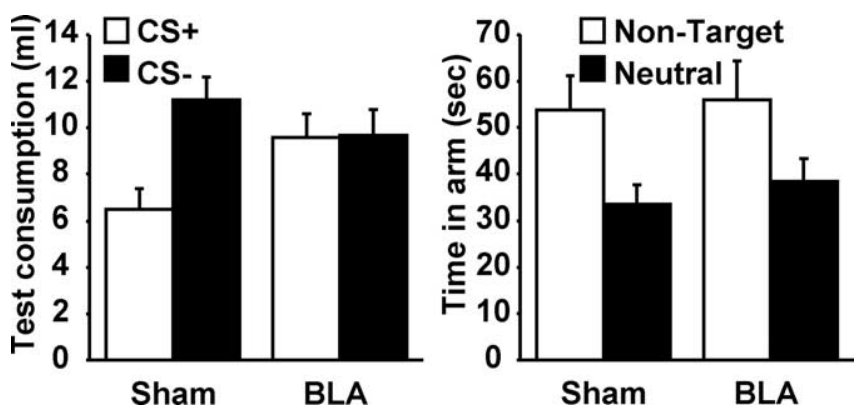

Figure 3. Left, Mean consumption (with SEM) of the $C S+$ and $C S-$ for both sham- and BLA-lesioned rats in experiment 1B. Right, Mean time (with SEM) spent in the nontarget and neutral arms of the $Y$ maze during place-preference testing. The nontarget arm had been paired previously with a nutrient solution, whereas the neutral arm had been paired previously with water.

\section{Discussion}

Experiment 1A confirms that BLA-lesioned rats do not show impaired conditioned taste aversion. Nor were they impaired on sensory preconditioning. That is, after two neutral flavors were paired, subsequently creating an aversion to one of them also reduced consumption of the flavor paired previously with it. Thus, they showed no deficit in a task requiring them to respond according to the current motivational value of a retrieved stimulus representation. This replicates Blundell et al. (2003) and confirms that BLA lesions do not prevent the formation of associations between neutral events. Experiment $1 \mathrm{~B}$ examined whether BLA lesioned rats would be impaired when they were required them to learn a new association based on the retrieved representation of a motivationally significant event. Rats were presented with two flavored nutrient solutions, each paired with a different context CS (distinctive arms of a Y maze). Placing rats in one context activated the representation of the flavored solution presented previously in it. The rats were then made ill by $\mathrm{LiCl}$ injection so that the activated representation of the flavored nutrient solution was paired with illness, producing a representation mediated taste aversion for that solution. This occurred in sham-, but not BLA-lesioned rats. However, both groups learned to prefer the arm paired with the nondevalued US over a third arm paired previously with water.

Comparing the designs of experiment $1 \mathrm{~A}$, sensory preconditioning, and experiment $1 \mathrm{~B}$, representation-mediated conditioning, reveals that their surface structure is quite similar: both begin by pairing two cues, one of which is then presented alone along with illness created by $\mathrm{LiCl}$ administration. In sensory precondi- tioning, flavor A was paired with flavor $\mathrm{X}$, which was then devalued with LiCl leading to a subsequent rejection of flavor A in both BLA- and sham-lesioned rats. In mediated conditioning, a context CS was paired with a flavored nutrient solution, rats were then made ill with $\mathrm{LiCl}$ after being exposed to the context CS, which resulted in a rejection of the flavored solution presented previously in that context in sham-, but not BLA-lesioned rats. It is not simply that BLA-lesioned rats had learned nothing during pairings of the context CS and nutrient solution because they subsequently showed place preferences based on these pairings. Thus, it is unlikely that the differences between experiments $1 \mathrm{~A}$ and $1 \mathrm{~B}$ are related to the ease with which BLA-lesioned animals formed associations between flavored and contextual cues. Furthermore, because the critical test in both experiments was of the relative preference for sucrose and maltodextrin, the differences cannot be related to the discriminability of test stimuli.

Given these seemingly contrasting results, it is worth examining the associative mechanisms that underpin performance in sensory preconditioning and mediated conditioning, respectively. Sensory preconditioning relies on formation of neutral stimulus 1 (S1)-S2 associations in initial training, and subsequently learning that the neutral $\mathrm{S} 2$ was devalued by direct pairing with illness. Rejection of $S 1$ on test relies on its ability to retrieve the representation of the now devalued S2; this is supported by the fact that if the devaluation of $\mathrm{S} 2$ is reduced by extinction then the rejection of $\mathrm{S} 1$ is correspondingly reduced (Rizley and Rescorla, 1972). Mediated conditioning also relies on the formation of an association between two stimuli such that presentation of one will activate the representation of the other (test) stimulus. While the representation of the test stimulus is active (in its absence), a devaluing event is presented, forming an association between the representations of the test stimulus and the devaluing event. Responding to the test stimulus is not related to responding to the stimulus which had previously been paired with it (Holland, 1990; Ward-Robinson and Hall, 1998; Dwyer, 1999; 2001). Here, mediated conditioning depends on the context CS retrieving a full sensory representation of the flavored nutrient solution that had been presented previously in that context, and it is the pairing of this representation with illness that creates a direct aversion to that solution.

A clear explanation for the contrasting results of experiments $1 \mathrm{~A}$ and $1 \mathrm{~B}$ can be found in the nature of the representations retrieved in each experiment. Recent work (Blundell et al., 2001; 2003; Killcross and Blundell, 2002; Balleine at al., 2003) suggests that the BLA plays a specific role in the representation of the unique sensory aspects of motivationally significant stimuli. According to this account, animals with BLA lesions will from an impoverished representation of significant stimuli that excludes their specific sensory qualities and includes only their generalized motivational properties (Konorski, 1967; Hall, 1996). Thus, when a CS is paired with a motivationally significant stimulus, subsequent presentation of the CS alone will activate only a generalized motivational system without representing the sensory qualities of the reinforcer which distinguish it from other, similarly valenced, stimuli. The acquisition of first-order Pavlovian (e.g., experiments 1A and 1B) (Setlow et al., 2002; Blundell et al., 2003) and instrumental conditioning (Blundell et al., 2001; Balleine et al., 2003) supports the idea that some general reinforcement process is intact in BLA-lesioned animals. Although a general reinforcement process can support first-order conditioning, it is not sufficient to support additional learning about the precise reinforcer that originally gave rise to it, because the representation retrieved does not include specific sensory features of the 
motivational stimulus. In experiment $1 \mathrm{~B}$, the motivational significance of sucrose and maltodextrin (evidenced by their ability to support place preferences) would have obliged BLA-based processing. Thus, when BLA-lesioned animals were exposed to the contextual CS, only a general motivational representation would have been retrieved. Even if this representation was associated with illness, it would not be sufficient to support a selective rejection of either sucrose or maltodextrin.

Crucially, this account does not state that the BLA underpins learning about sensory aspects of events in all situations, only those involving motivationally significant stimuli. Thus, in experiment $1 \mathrm{~A}$, in which the nutrient content of dilute sucrose and maltodextrin are of negligible motivational importance in the absence of food deprivation and the possibility of representing any motivational importance of fluid per se would be minimized by the extensive previous exposure to water, lesions of the BLA are expected to be without effect. Indeed, Blundell et al. (2003) argued that the representation of a neutral S1-S2 association is not dependent on the BLA (for a possible site for such associations, see Nicholson and Freeman, 2000). Thus, sensory preconditioning that relies on representation of the neutral S1-S2 association and the devaluation of $\mathrm{S} 2$ by pairing with $\mathrm{LiCl}$ is unaffected by lesions of the BLA because neither process relies on the representation of the sensory aspects of a motivationally significant stimulus.

The different associative structures of sensory preconditioning and mediated conditioning highlight the fact that the crucial representations in experiment $1 \mathrm{~B}$ were of the sensory aspects of motivationally significant stimuli, whereas in experiment $1 \mathrm{~A}$ the crucial representations were of neutral stimuli. There is debate regarding the rules governing associations involving the representations of absent stimuli (Dwyer, 2003), which raises the possibility that BLA- and sham-lesioned animals may differ with respect to learning about the associatively activated representation of any stimulus (rather than just motivationally significant ones). However, there has been no previous suggestion that BLA lesions impair the formation of associations between stimulus representations per se. Indeed, the demonstration that animals with lesions of the BLA are able to learn directly conditioned taste aversions (experiment 1A) (Dunn and Everitt, 1988; Blundell et al., 2003) indicates that they are able to learn an association between the representation of a flavor and illness. Alternatively, the fact that experiment $1 \mathrm{~B}$ relied on context-taste associations, whereas experiment $1 \mathrm{~A}$ relied on taste-taste associations, raises the possibility that the failure of mediated conditioning was not caused by BLA lesions preventing sensory associations to rewarding stimuli, but was instead the result of lesions interfering with associations between multimodal sensory cues (Hatfield et al., 1992; Hatfield and Gallagher, 1995). However, the fact that BLAlesioned animals were able to learn a place preference in experiment $1 \mathrm{~B}$ suggests that they were not prevented from forming an association between the context and some representation of the nutrient solution presented in it. In addition, Setlow et al. (2002) suggested that auditory-visual associations survived BLA lesions, and we have observed intact odor-taste learning in both appetitive and aversive versions of a sensory preconditioning task (G. K. Bailey, P. Blundell, M. Symonds, G. Hall, and S. Killcross, unpublished observations).

In summary, we have demonstrated that BLA-lesioned rats are unable to use the retrieved representation of a motivationally significant stimulus to support additional learning. Combined with deficits in performance based on the retrieval of sensory specific representations of particular reinforcers such as the facilitatory differential outcomes effect and reinforcer specific Pavlovian to instrumental transfer (Blundell et al., 2001) or outcome devaluation by sensory-specific satiety and the degradation of action-outcome contingencies with multiple outcomes (Balleine et al., 2003), our results suggest that lesions of the BLA do not simply impair responding based on the sensory aspects of reinforcers, but that they prevent the representation of the sensory aspects of motivationally significant stimuli per se.

\section{References}

Abramoff MD, Magelhaes PJ, Ram SJ (2004) Image processing with ImageJ. Biophotonics Int 11:36-42.

Balleine BW, Killcross AS, Dickinson A (2003) The effect of lesions of the basolateral amygdala on instrumental conditioning. J Neurosci 23:666-675.

Blundell P, Hall G, Killcross S (2001) Lesions of the basolateral amygdala disrupt selective aspects of reinforcer representation in rats. J Neurosci 21:9018-9026.

Blundell P, Hall G, Killcross S (2003) Preserved sensitivity to outcome value after lesions of the basolateral amygdala. J Neurosci 23:7702-7709.

Corbit LH, Balleine BW (2005) Double dissociation of basolateral and central amygdala lesions on the general and outcome-specific forms of pavlovian- instrumental transfer. J Neurosci 25:962-970.

Dunn LT, Everitt BJ (1988) Double dissociations of the effects of amygdala and insular cortex lesions on conditioned taste-aversion, passiveavoidance, and neophobia in the rat using the excitotoxin ibotenic acid. Behav Neurosci 102:3-23.

Dwyer DM (1999) Retrospective revaluation or mediated conditioning? The effect of different reinforcers. Q J Exp Psychol B 52:289-306.

Dwyer DM (2001) Mediated conditioning and retrospective revaluation with LiCl then flavor pairings. Q J Exp Psychol B 54:145-165.

Dwyer DM (2003) Learning about cues in their absence: evidence from flavor preferences and aversions. Q J Exp Psychol B 56:56-67.

Hall G (1996) Learning about associatively activated stimulus representations: Implications for acquired equivalence and perceptual learning Anim Learn Behav 24:233-255.

Hatfield T, Gallagher M (1995) Taste-potentiated odor conditioning-impairment produced by infusion of an N-methyl-D-aspartate antagonist into basolateral amygdala. Behav Neurosci 109:663-668.

Hatfield T, Graham PW, Gallagher M (1992) Taste-potentiated odor aversion learning-role of the amygdaloid basolateral complex and central nucleus. Behav Neurosci 106:286-293.

Hatfield T, Han JS, Conley M, Gallagher M, Holland P (1996) Neurotoxic lesions of basolateral, but not central, amygdala interfere with pavlovian second-order conditioning and reinforcer devaluation effects. J Neurosci 16:5256-5265.

Holland PC (1990) Event representation in Pavlovian conditioning: image and action. Cognition 37:105-131.

Killcross AS, Blundell P (2002) Associative representations of emotionally significant outcomes. In: Emotional cognition (Moore SC, Oaksford M, eds), pp 35-73. Amsterdam; Philadelphia: John Benjamins.

Konorski J (1967) Integrative activity of the brain. Chicago: University of Chicago.

Morris R, Frey S, Kasambira T, Petrides M (1999) Ibotenic acid lesions of the basolateral, but not the central, amygdala interfere with conditioned taste aversion: evidence from a combined behavioral and anatomical tract-tracing investigation. Behav Neurosci 113:291-302.

Nicholson DA, Freeman JH (2000) Lesions of the perirhinal cortex impair sensory preconditioning in rats. Behav Brain Res 112:69-75.

Paxinos G, Watson C (1998) The rat brain in stereotaxic coordinates. San Diego: Academic.

Rizley RC, Rescorla RA (1972) Associations in second-order conditioning and sensory preconditioning. J Comp Physiol Psychol 81:1-11.

Setlow B, Gallagher M, Holland PC (2002) The basolateral complex of the amygdala is necessary for acquisition but not expression of CS motivational value in appetitive Pavlovian second-order conditioning. Eur J Neurosci 15:1841-1853.

Ward-Robinson J, Hall G (1998) Backward sensory preconditioning when reinforcement is delayed. Q J Exp Psychol B 51:349-362. 